\title{
Multi-Physics Modeling for Laser Micro-Transfer Printing Delamination
}

\author{
Ala’a M. Al-okaily ${ }^{\mathrm{a}}$, Placid M. Ferreira ${ }^{\mathrm{a}, *}$
}

${ }^{\mathrm{a}}$ Department of Mechanical Science and Engineering, University of Illinois at Urbana-Champaign, 1206

W. Green Street, Urbana, IL, 61801, USA

\section{Email Addresses:}

Ala’a M. Al-okaily: alokail2@illinois.edu

Placid M. Ferreira: pferreir@illinois.edu

*To whom correspondence should be addressed: pferreir@illinois.edu, +1-217-333-0639. 


\begin{abstract}
Micro-transfer printing technology is rapidly emerging as an effective pathway for large-scale heterogeneous materials integration. In its basic embodiment, micro-transfer printing is used to deterministically transfer and micro-assemble prefabricated structures/devices, referred to as "ink," from a donor substrate to a receiving substrate using a viscoelastic elastomer stamp, usually made out of polydimethylsiloxane (PDMS). Laser Micro Transfer Printing (LMTP) is a laser-driven non-contact variant of the process that makes it independent of the receiving substrate's properties, geometry, and preparation. In this paper, an opto-thermo-mechanical model is developed to understand how the laser beam energy is converted to thermally-induced strains around the ink-stamp interface to initiate the ink delamination process. The optothermo-mechanical model is developed based on decoupling the optical absorption physics from the thermo-mechanical model physics. An optical absorption model for the laser beam energy absorbed by the ink is first developed and verified experimentally to estimate the heating rates of the ink-stamp system, which in turn are used as an input for a couple thermomechanical Finite Element Analysis (FEA) model. Further, high speed camera recordings for LMTP delamination are used to calibrate the thermo-mechanical model and verify its predictions. Besides providing a fundamental understanding of the delamination mechanism and the LMTP process capabilities, the developed opto-thermo-mechanical model is useful in selecting process parameters (laser pulse duration, stand-off distance), estimating the ink-stamp temperature rise during the LMTP process, and quantifying and decomposing the stresses at the ink-stamp interface to its main sources (Coefficient of Thermal Expansion (CTE) mismatch and thermal gradient strains).
\end{abstract}

\title{
Keywords
}

Micro-Transfer Printing; Laser Micro-Assembly; Flexible Electronics; Laser-Driven Delamination; Fracture Mechanics; Multi-Physics Modeling.

\section{Introduction}

Micro-transfer printing, due to its simplicity, accuracy, repeatability, and large-area substrate printing capabilities, is rapidly emerging as an effective method to enable large-scale heterogeneous materials integration (examples shown in Figure 1 [1-6]). In its basic embodiment, micro-transfer printing is used to deterministically transfer and micro-assemble prefabricated structures/devices, referred to as "ink," from a donor substrate to a receiving substrate. The mechanism of ink pick-up and release is based on modulating the adhesion energy kinetically between the ink and a viscoelastic elastomer 
stamp [7], usually made out of polydimethylsiloxane (PDMS). To enhance and extend the transfer printing technology capabilities and performance, several contact mode variants of the process have been introduced by modifying the stamp's geometry (patterned stamps [8], pedestal-shaped stamps [9], and microtipped stamps [1]) or enhancing the transfer mechanism (shear-enhanced [10] and fluidic-chamber actuated [11]). Recently, Laser Micro Transfer Printing (LMTP) has been introduced [12] [13] to enable non-contact printing, allowing the transfer printing performance to become independent of the receiving substrate's properties, preparation, and geometry (examples shown in Figure 2). The LMTP printing cycle starts by selectively picking-up ink from a donor substrate with a patterned PDMS stamp (Figure 3-a and 3-b), then positioning the ink above a desired location on the receiver substrate at a stand-off height (Figure 3-c), and then pulsing a laser to drive the release of the ink from the stamp (Figure 3-d). Since the ink pick-up and transfer steps are similar to microtransfer printing, the LMTP process is different in terms of the release mechanism that is based on generating laser-induced thermo-mechanical stress at the ink-stamp interface. A NIR laser beam ( $805 \mathrm{~nm}$ wavelength with $\sim 700 \mu \mathrm{m}$ spot size) is transmitted through the glass stamp holder and the PDMS stamp and absorbed by the ink, usually Si or GaAs. The laser beam power absorbed by the ink heats the ink which, in turn, transfers heat to the PDMS stamp, raising the ink-stamp interface temperature. Due to the low thermal conductivity of the PDMS, a localized hot zone is developed in the PDMS in the vicinity of the ink-stamp interfaces. The PDMS in this zone expands because of its large CTE $\left(310 \mathrm{ppm} /{ }^{\circ} \mathrm{C}\right)$. Constrained by the silicon ink (CTE $\left.2.6 \mathrm{ppm} /{ }^{\circ} \mathrm{C}\right)$ and the surrounding unheated PDMS, this expansion is accommodated by the development of a curvature or bulge at the contact interface. The curvature gives a rise to a bending moment that stresses the ink-stamp interface normal to the interface direction (opening mode) and along the interface (shear mode). Further, the sharp thermal gradient at the ink-stamp interface edges loads the ink-stamp interface in both directions (open and shear modes). Once the stored strain energy, measured by the Energy Release Rate (ERR), at the interface reaches the work of adhesion of the inkstamp interface $W$, the delamination crack, at the perimeter of the ink, propagates inwards to release the ink from the PDMS stamp.

Our previous work [14], using high-speed camera observations, studied the delamination time, the time interval from the start of the laser pulse to the start of the delamination of the ink, for square silicon ink. Li et. al [15] used an analytical axisymmetric thermo-mechanical model to estimate the ERR for the LMTP process based on the CTE mismatch strains. Using such an approach, the ERR is then used to estimate the delamination time based on Griffith's criterion for a given work of adhesion of the ink-stamp interface. In this paper, the focus will be on developing a coupled thermo-mechanical Finite Element Analysis (FEA) model to understand and decompose the effect of both, the CTE mismatch at the interface, and the 
thermal gradient strains within the PDMS stamp to study and predict the LMTP delamination process behavior at different printing conditions.

\section{Experimental Setup}

A laser printer is designed with a print head that uses an $805 \mathrm{~nm}$ wavelength, electronically pulsed, continuous wave laser diode source to generate laser pulses with a minimum pulse duration of $1 \mathrm{msec}$. The laser beam power is controlled by varying the diode current to generate variable beam power in the range of $0-30 \mathrm{~W}$. The laser beam is transmitted through a $200 \mu \mathrm{m}$ diameter optical cable to the print head, folded through a 90 degree angle, and focused at the plane of the ink-stamp interface. A dichroic mirror that reflects at $805 \mathrm{~nm}$ but transmits in the visible band allows imaging during the printing process, as shown in Figure 4. Using numerically controlled XYZ stages with an accuracy of $\pm 0.5 \mu \mathrm{m}$ and a bidirectional repeatability of $\pm 0.3 \mu \mathrm{m}$ over $100 \mathrm{~mm}$ travel range, the printer (see Figure 5) has the capability to precisely position the print head relative to the donor and receiving substrates. Further, A LabVIEW ${ }^{\circledR}$ (National Instruments) software interface is designed to automate the steps in the LMTP printing cycle. More details on the printer design and components can be found in [14].

\section{LMTP Process Modeling}

Understanding the mechanism of delamination during the LMTP process requires integrating the effects of multiple physical phenomena involved in the process. Therefore, the modeling approach is based on first developing an optical absorption model under the assumption that absorption during the LMTP process is decoupled from the thermo-mechanical physics. The optical absorption model is used to estimate the heating rate of the ink during the LMTP process which, in turn, is used as an input to the coupled thermo-mechanical FEA model. In our previous paper on the delamination mechanism [14], a transient coupled FEA thermo-mechanical model was developed to estimate the stresses, strains, temperature gradient, and temperature fields during the LMTP process using COMSOL Multiphysics ${ }^{\circledR}$ (COMSOL, Inc). However, to extend this to the onset of delamination; so as to understand, control, and predict the delamination process behavior, the fracture mechanics quantities such as the ERR and the Stress Intensity Factors (SIFs) are essential. Therefore, a coupled dynamic thermomechanical FEA model is developed in $\mathrm{ABAQUS}^{\circledR}$ (ABAQUS Inc.) where, by introducing a small crack at the ink-stamp interface edge, one can collect the ERR and the SIFs during the LMTP process. The end goal of such a model is to understand how different thermal strain components (CTE mismatch and thermal gradient within the stamp's post) affect the 
printing process at different laser beam powers and different critical dimensions (in this paper, thickness) of the ink. Such predictions will help in planning the laser pulse duration required to print different inks, planning the stand-off distance to avoid crack closure due to ink-receiver substrate collision, estimate the temperatures reached by the ink during the printing process, quantify and decompose the thermally-induced strains at the ink-stamp interface, and understand the process capabilities and limitations.

\subsection{Optical Absorption Model}

As a result of optical absorption, the laser beam intensity transmitted through the ink drops exponentially as described by the Beer-Lambert law [16]. Therefore, once the laser beam enters the ink material, it is transmitted through the material, undergoing some transmission losses. When the beam encounters a boundary, a portion is internally reflected. This is repeated as the beam consecutively encounters the top and bottom surfaces of the ink. The total power absorbed by the ink $\left(P_{b}\right)$ in all passes is the summation of the absorption in each passage. Therefore,

$$
F_{b}=\Sigma ?
$$

where $n$ is the number of times the beam passes through the ink. If $R_{T}$ is the reflectivity of the top ink interface, $R_{B}$ the reflectivity of the bottom ink interface, $\alpha$ is the absorption coefficient for the ink material at the wavelength of the laser radiation, $h_{s}$ is the ink thickness, and $P_{a}$ is the power of laser beam incident on the ink, then the power absorbed in the $i^{\text {th }}$ pass $\left(P_{i}\right)$ is given by (for more details, see [14]):

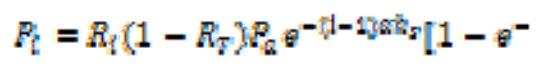

where

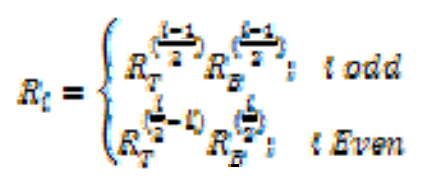

Since the laser beam energy profile is mesa-shaped, with a flat-top that can circumscribe a square with a $400 \mu \mathrm{m}$ side and Gaussian-like side walls shapes, the heating for $200 \times 200 \mu \mathrm{m}$ inks can be considered uniform. The model is then used to estimate the laser beam power absorbed by $200 \times 200 \mu \mathrm{m}$ square silicon inks (with thicknesses of $3,10,30,50 \mu \mathrm{m}$ ) at three different laser diode current $(10,15$, and $20 \mathrm{~A}$ which are equivalent to laser beam power of $3.268,6.587$, and $9.956 \mathrm{~W}$, respectively). The measurements of the power absorbed by the inks is performed using a $5.2 \mathrm{~W}$ maximum multi-wavelength power meter (PM100D; Thorlabs Inc.) by taking the average of the difference in power arriving at the meter with and without 
the ink in its path and accounting for reflective losses. A number of experiments were conducted to measure the laser beam power absorbed by $200 \times 200 \mu \mathrm{m}$ square silicon inks with four different thicknesses $(3,10,30,50 \mu \mathrm{m})$ at current settings of 10 and $15 \mathrm{~A}$ (20 A produces a power level above the measurement range of the meter). As shown in Figure 7, the measured powers absorbed by the silicon inks agree strongly with the model's predicted trends and values. Assuming uniform heating of the ink (justified by previous results from FEA simulations that show that there is no temperature gradient within the ink due to the high thermal conductivity of silicon), the estimated and measured heating rates are shown in Figure 8. Larger ink thicknesses usually exhibit lower heating rates due to the large ink thermal mass even though the amount of power absorbed by thicker inks is higher. The uncertainty in the measurements of the heating rates are larger for thinner inks since the difference between the two laser pulses energy (with and without the ink) is low compared to measured pulse energy (low $\mathrm{S} / \mathrm{N}$ ratio).

\subsection{Delamination Time Experiments}

The effect of varying $200 \times 200 \mu \mathrm{m}$ square silicon inks' thicknesses $(3,10,30,50 \mu \mathrm{m})$ and laser beam power levels $(10$, $15,20 \mathrm{~A}$ ) has been investigated to estimate the pulse duration of the laser required to start the delamination process. Figure 9 shows the trends for the delamination time, obtained from the experiments using a high speed camera at frames rate of 8000 frame/sec with a PDMS stamp having a $400 \times 400 \times 50 \mu \mathrm{m}$ post. The results show that the thinner the ink (from 3 to $50 \mu \mathrm{m}$ thickness) or the higher the power level (from $10 \mathrm{~A}$ to $20 \mathrm{~A}$ power levels), the shorter the delamination time. The results also show insignificant difference in printing ink with 3 and $10 \mu \mathrm{m}$ thicknesses. For most of the printing conditions, except at 30 and $50 \mu \mathrm{m}$ thickness at $10 \mathrm{~A}$ current level of the laser, the delamination, once started, is completed within one frame $(<0.125$ msec.) of the high speed camera. Since the heating rates for these two cases (30 and $50 \mu \mathrm{m}$ thicknesses at 10 A power level) are slow, the delamination process can be observed to take $0.625-0.75 \mathrm{msec}$. (equivalent to $0.133-0.16 \mathrm{~m} / \mathrm{sec}$. delamination propagation speed) and $0.75-0.875 \mathrm{msec}$. (equivalent to $0.114-0.133 \mathrm{~m} / \mathrm{sec}$. delamination propagation speed), respectively. Further, the experiments show that for all ink thicknesses, the delamination process is stable, where the crack propagation progress can be terminated and reversed (crack closing) by having the laser pulse duration slightly less than the delamination time. Such behavior occurs because, while the discontinuity at the edge of the ink is sufficient to initiate the delamination crack, a continuous supply of thermal energy by the laser beam is required to maintain the ERR at the interface above the work of adhesion of the interface to drive the delamination to completion. 


\subsection{Thermo-Mechanical Model Development}

A coupled 3D transient thermo-mechanical model is developed in ABAQUS using reduced integration quadratic coupled temperature-displacement elements accounting for the large material deformation. The model input is the uniform heating rate estimated from the optical absorption model in section 3.1 as a body heat source in the silicon ink. The model assumes linear elastic material behavior where the material properties are listed in [14]. Since modeling the backing layer of the PDMS stamp thickness does not affect the output results if the post height is sufficiently large, a large PDMS post height of $100 \mu \mathrm{m}$ is used for these simulations. A displacement boundary condition is enforced at the end of the PDMS post to restrict the translational and the rotational movement of the elements' nodes at the post end. At the ink-stamp interface edges, a $2 \mu \mathrm{m}$ crack is introduced to collect the ERR and the SIFs based on the J-integral method and interaction integral method, respectively. To calibrate the model, a 3D model with $10 \mu \mathrm{m}$ global element size was utilized to simulate the printing of $200 \times 200 \times 50 \mu \mathrm{m}$ ink up to the delamination start time $(9.75 \mathrm{msec}$.) at $10 \mathrm{~A}$ current level. Using the same ink, the maximum post's lateral deformation was experimentally measured by imaging the ink-stamp system from the top, using a high speed camera, as shown in Figure 10-a and Figure 10-b. The experimental results show that the maximum post lateral dimension at delamination point increases by $1.76 \%$ (equivalent to $L_{f}-L_{o}=7.05 \mu \mathrm{m}$ with $0.56 \mu \mathrm{m}$ pixel resolution) compared to $6.01 \mu \mathrm{m}$ estimated from the 3D model output (Figure 10-c). Therefore, one can conclude with reasonable confidence that the modeling approach (the power absorption and the FEA thermo-mechanical) is able to predict the LMTP optical-thermo-mechanical physics interaction and the process behavior up to the start of delamination with acceptable accuracy.

To ensure independence of mesh size, it would be necessary to evaluate finer meshes than the $10 \mu \mathrm{m}$ equally sized cubical elements for the 3D FEA model. However, the computational power and time grow exponentially with reducing mesh size. Therefore, we first evaluated the use of an equivalent axisymmetric model, a 3D cylindrical model with the same mesh size $(10 \mu \mathrm{m})$ and equivalent areas (ink diameter $225 \mu \mathrm{m}$ and post diameter $450 \mu \mathrm{m}$ ). The results for square 3D model, cylindrical 3D model, and the axisymmetric at different global meshing sizes models are shown in Table 1 . The results indicate that the cylindrical or the axisymmetric model can be used to reasonably approximate the printing of the square inks, neglecting the corners effect. Further, from the axisymmetric model results, the stresses, strains, and strain energy density fields are mesh-dependent due to the geometry singularity at the interface edges. On the other hand, the primary element variables (displacements and temperature) and the fracture mechanics quantities (ERR, SIFs) are mesh-independent. The values of these mesh independent variables converge if the global mesh size is around $1 \mu \mathrm{m}$. Figure 11 shows the ERR as a function of the laser pulse time for the different models in printing $200 \times 200 \times 3 \mu \mathrm{m}$ ink at the $10 \mathrm{~A}$ current level. The ERR for 
the 3D square model is higher at the corners compared to the other location at the interface edges. Moreover, the 3D cylindrical model and the axisymmetric models have ERRs in between the two values of EERs at the corner and the edge from the 3D square model, being closer to the ERR at the edge. Since the ERR has to equal the work of the adhesion at each individual location at the crack, the axisymmetric model is expected to overestimate the ERR values at the square ink edge. In experiments involving printing equivalent area square and round inks, round inks require less time to print (4-10\%) compared to square inks. This agrees with the axisymmetric model's estimate of a higher ERR at the interface edge. In summary, the axisymmetric model with $1 \mu \mathrm{m}$ meshing element size can be used to estimate the printing of square inks using the LMTP process.

\subsection{Axisymmetric Model Results}

Using the axisymmetric model, the fields for the thermo-mechanical variables at delamination start time (measured in section 3.2) are shown in Figure 12. The temperature field (Figure 12-a) shows no thermal gradient within the silicon ink and a large thermal gradient within the PDMS post. After $50 \mu \mathrm{m}$ from the ink-stamp interface, the thermal field becomes uniform (room temperature). The results also show that the heat flux from the silicon ink to the PDMS post is almost uniform at the interface except around the crack edge. Figures 12-c and 12-d show the displacement and the axial displacement fields indicating that the silicon ink, because of its high stiffness relative to PDMS, does not exhibit large deformations. Further, Figure 12-e and 12-f show the mode I (opening mode normal to interface direction) strain and stress fields, respectively, while Figure 12-g and 12-h show the mode II (shearing mode along the interface direction) strain and stress fields, respectively. Since the traction at the interface is equal, the stress fields in mode I and mode II direction are continuous and smooth, while the strains fields in both directions are discontinuous, especially at the interface edge, due to the large mismatch in Young's modulus at the interface. Thus, the locations of maximum stress, strain, and strain energy density points are always at the interface edge in the axisymmetric model (at the corners for 3D square model).

Figure 13-a, 13-b, and 13-c show the model estimations for the interface temperature as a function of time, essentially the silicon ink temperature, for different ink thicknesses up to the delamination time (measured in section 3.2) at $10 \mathrm{~A}, 15 \mathrm{~A}$, and 20 A laser current, respectively. The curves show that regardless of the current levels, thin inks (3 and $10 \mu \mathrm{m})$ print at roughly the same delamination time and at a lower temperature compared to thick inks (30 and $50 \mu \mathrm{m})$. Further, thick inks print almost at roughly the same interface temperature. The rate of change of interface temperature is higher for thin inks ( 3 and $10 \mu \mathrm{m}$ ) compared to thick inks (30 and $50 \mu \mathrm{m}$ ) and increases with laser current. Finally, under all conditions the rate of 
temperature change is highest for $10 \mu \mathrm{m}$ inks, suggesting that at these power levels, the $10 \mu \mathrm{m}$ inks thickness give the best balance between the thermal energy stored in the ink and that transferred to the PDMS stamp.

During printing, when inks with different thicknesses are printed using the same stamp dimensions, the heat flux from the silicon ink to the PDMS can be considered as a measure for both: the rate at which thermal energy is converted into strain energy, and its localization around the ink-stamp interface. Therefore, heat flux output from the FEA model (see Figure 14-a, 14-b, and 14-c for $10 \mathrm{~A}, 15 \mathrm{~A}$, and $20 \mathrm{~A}$, respectively) suggest that time constant for heat flux depends on the ink thickness (longer time constant for thicker inks). The heat flux graphs in Figure 14 show that the initial heat flux is highest for thin inks (low ink heat capacity). Further, thin inks $(3 \mu \mathrm{m}$ and $10 \mu \mathrm{m})$ almost reach a steady state heat flux within the delamination time, while the heat fluxes for thick inks $(30$ and $50 \mu \mathrm{m})$ are still increasing when delamination occurs at all laser beam power levels. The high heat flux for thinner inks at the start of the heating cycle (fast heating for PDMS) leads to higher temperature gradients in the PDMS, in turn, leading to a more highly stressed interface and a shorter delamination time. For thicker inks, the heat flux is lower at the start of the heating cycle (slow PDMS heating).

The ERR at the crack tip was estimated using the J-integral method for the printing of different ink thicknesses and different laser current levels (see Figure 15- a, 15- b, and 15-c for $10 \mathrm{~A}, 15 \mathrm{~A}$, and $20 \mathrm{~A}$, respectively). The results show that regardless of the laser current level, the ERR at delamination point for each ink thickness is almost constant, even though different inks require different delamination times at different laser current levels. Based on Griffith's criterion, the critical ERR values $\left(G_{c}\right)$ at the delamination point should equal the work of adhesion for Silicon-PDMS interface (interface property reported to be $W=0.05-0.151 \mathrm{~J} / \mathrm{m}^{2}$ at room temperature [1] [17]). However, the results clearly indicate that the critical ERR depends on the ink thickness, which is not expected. A plausible reason for this follows from the fact that heating of the inkstamp stack system for the same ink thickness at different power levels generated almost the same interface temperature rise that leads to approximately the same ERR at the delamination point. Therefore, the delamination process is dominated by the temperature of the interface. For thick inks (30 and $50 \mu \mathrm{m}$ ), which print at higher temperatures, the work of adhesion between silicon and PDMS may be higher and constant, causing the ERR at the delamination point to be almost the same. For thinner inks, where the delamination temperatures are in the 200 degree $\mathrm{C}$ range, the work of adhesion of the interface is still changing and hence, one sees that the ERR at delamination is different for thin inks (ERR at delamination for $3 \mu \mathrm{m}$ inks is about half of that for $10 \mu \mathrm{m}$ inks). It could also be that the higher temperatures encountered for thicker inks result in temperature-dependent changes in the PDMS viscoelastic properties. Our models, being primarily elastic in nature, do not account for these effects and therefore overestimate the ERR at delamination. These results from the FEA model, along with 
huge plastic deformation in the PDMS post at interface edges after printing thick inks, imply that the ERR at delamination varies as a function of the PDMS temperature. Further, videos captured using the high speed camera while printing thick inks, especially $30 \mu \mathrm{m}$ thickness, show that PDMS post undergoing cyclic deformation around the interface edges during the laser heating. Such cyclic deformation suggests a periodic release of the stored energy in the PDMS at high temperature due to plastic flow before eventually overcoming the interface's work of adhesion, which is also temperature-dependent. Because the model does not account for such plastic flow and temperature-dependent properties of the stamp-ink interface, it computes a higher value for ERR at delamination. However, the model should be adequate for estimating the LMTP process behavior for thin inks (i.e. $200 \times 200 \times 3 \mu \mathrm{m}$ ink) where the maximum temperature rise is less than $200{ }^{\circ} \mathrm{C}$.

Figure 16-a and 16-b show the mode I SIF $\left(K_{I}\right)$ and mode II SIF $\left(K_{I I}\right)$ for printing different inks thicknesses at 10 A current level. The results show that the LMTP delamination process is a mixed-mode fracture problem where $K_{I}$ has positive value (tensile load in mode I trying to open the interface) and $K_{I I}$ has negative value (negative shear load in mode II trying to squeeze the interface toward interface center). In linear fracture mechanics, the ERR value $G=\frac{\frac{1}{S}}{\mathbb{S}}\left(K^{n}+\right)($ where is the interface equivalent Young's modulus and it is a function of the ink and the stamp Young's modulus

$\tilde{E}=2 E_{\operatorname{lnk}} E_{s t a m p} /\left(E_{\ln k}+E_{s t a s}\right)$ is a composition of the SIFs squares at the crack tip. The SIFs trends are similar to ERR where thin inks prints at lower SIFs, in mode I and mode II, compared to thick inks, while the mode II contribution on building the ERR at the interface is almost two times higher than mode I for all ink thicknesses. Further, SIFs rise rates as function of laser beam pulsing time are higher for the inks that have higher interface temperature rise rates. Furthermore, evaluating the SIFs in both fracture modes for different inks thickness as a function of the interface temperature shows that for all ink thicknesses build the same SIF at given interface temperature in both fracture mode directions. This indicates that SIFs values, in both fracture modes, are dependent on interface temperature and independent of the inks geometry (difference in SIFs as function of laser pulsing time are due to different heating rates).

\section{Thermal Strain Energy Components}

Using the model developed in the previous section 3, one can decompose the SIFs into two components based on the sources of thermal strains: CTE mismatch between the ink and the stamp, and thermal gradient within the PDMS. To obtain the effect of the thermal gradient, the model can be evaluated with both the materials having the same CTE (i.e., 310 $\mathrm{ppm} /{ }^{\circ} \mathrm{C}$ ). On the other hand, uninform heating for the ink-stamp stack will eliminate the effects of the thermal gradient within 
the PDMS post. However, this will introduce thermal strains arising from the boundary condition, restricting the movement of the uniformly deformed PDMS stamp post. This boundary effect is an artifact, since in actual printing, the heat-affected zone is confined to a few microns $(<50 \mu \mathrm{m})$ from the interface because of the low thermal conductivity of PDMS. To eliminate this boundary effect, a third model is constructed with matched CTEs and uniform heating. The SIFs along both modes from the three simulations are combined (the SIF for each mode from the matching CTE model is added to that from the uniform heating model and that from the model with matching CTE and uniform heating is subtracted from the result). The linear combination of the three virtual simulations are compared to the original model output to produce an exact match (see Figure 17 -a and $17-\mathrm{b}$ for $200 \times 200 \times 3 \mu \mathrm{m}$ ink at $10 \mathrm{~A}$ current level), suggesting that the assumption of linearity is valid and that the relative contributions of CTE mismatch and thermal gradients can be studied in this manner.

This approach is then used to study printing of inks with different thicknesses at $10 \mathrm{~A}$ current (the same results hold for 15 and 20 A current level) as shown in Figure 18 (18-a for mode I SIFs $K_{I}$ and 18-b for mode II SIFs $K_{I I}$ ). The results show that for the mode I SIF, the CTE mismatch and thermal gradient mechanical strains both act in the same direction generating tensile strains trying to open the crack tip at interface edge. In the mode II direction, however, the CTE mismatch introduces a negative shear load at the interface edges sequinning the interface while thermal gradient strains generate a positive shear load stretching the interface. Further, in both directions, the CTE mismatch strains have a linear relationship with the interface temperature while thermal gradient strains have nonlinear relationship with the interface temperature. Furthermore, in mode I, because of non-linear growth, at higher temperature $\left(>100{ }^{\circ} \mathrm{C}\right)$, the contribution of the thermal gradient in the PDMS to the accumulation of strain energy at the crack tip dominates that of the CTE mismatch for different ink thicknesses, indicating that it is bending moment form the CTE mismatch load, in mode I direction, is less effective compared to the PDMS bulging due to thermal gradients within the stamp. The thermal gradient effect is also slightly lower for thin inks (3 and $10 \mu \mathrm{m}$ ) in mode I direction. In mode II, the contribution of the CTE mismatch is always higher than that of the thermal gradient strains generated at all temperatures for all ink thicknesses. The results from this decomposition approach imply that the thermal gradient within the PDMS plays an important and a significant role during the LMTP process. Therefore, its high CTE coupled with its low thermal conductivity make PDMS a good stamp material for the LMTP process. Furthermore, this decomposing approach shows the axial stamp deformation and bulging are generated due to the thermal gradient strains (both disappear by uniformly heating of the ink-stamp simulations). 


\section{Conclusions}

An opto-thermo-mechanical model for the LMTP delamination process has been developed based on decoupling the optical absorption physics from the thermo-mechanical model physics. A series of experiments was conducted to verify the heating rate estimated from the optical absorption model and to measure the delamination time during the LMTP process for sets of $200 \times 200 \mu \mathrm{m}$ square silicon inks with varying the ink thickness. Both the heating rates from the optical absorption model and the delamination times form the high speed camera are used as inputs for coupled FEA thermo-mechanical model. The FEA model was calibrated based on the PDMS post lateral dimension deformation, which was experimentally measured using a high speed camera. The model was then simplified to an axisymmetric model and used to study the LMTP process mechanism. The study has shown that the LMTP process is a mixed-mode process (mode II more dominant) where the critical ERR and SIFs (in both modes) were of equal magnitude for printing the same ink thickness at different laser current levels. However, for printing different inks' thicknesses, the critical ERR and SIFs (in both modes) were dependent on the ink thickness. Given the higher temperatures encountered in printing thicker inks, it is possible that plastic strain in the PDMS at these temperatures relaxes the mismatch strains at the interface to produce the apparent high critical ERR computed for thick inks. This implies that the LMTP process is more efficient and well understood in case of printing thin inks $\left(h_{s}<10\right.$ $\mu \mathrm{m})$ where the model predictions for delamination time agree with the experimental values. The future work will focus on designing patterned stamps to achieve printing at lower energy and interface temperature by enhancing mode I loading (mode I SIFs is lower than mode II SIFs while the work of adhesion required to break the interface is high in mode I).

The ERR or SIFs at the edge of the interface where the delamination crack originates were decomposed into two components: one due to CTE mismatch and the other due to the thermal gradient within the PDMS. Both components are significant in both fracture directions (mode I and mode II) of growth of the delamination crack, suggesting that both, the high coefficient of thermal expansion and the low thermal conductivity of the PDMS are essential properties for it to function as a stamp material in the LMTP process.

\section{Acknowledgements}

The material presented here is based on work supported by the Center for Nanoscale Chemical-Electrical-Mechanical System (NanoCEMMS), a Nanoscale Science and Engineering Center sponsored by NSF under Award \# 0749028 (CMMI). It is also supported by the NSF under Award \# 1301336 (CMMI) and the Grayce-Wicall Gauthier Professorship. The authors would also like to thank Numair Ahmed and Bonjin Koo for their help with the experiments. 


\section{References}

[1] Kim S, Wu J, Carlson A, et al. Microstructured elastomeric surfaces with reversible adhesion and examples of their use in deterministic assembly by transfer printing. Proceedings of the National Academy of Sciences. 2010;107(40):17095-17100.

[2] Chanda D, Shigeta K, Gupta S, et al. Large-area flexible 3D optical negative index metamaterial formed by nanotransfer printing. Nature Nanotechnology. 2011;6(7):402-407.

[3] Kim H-s, Brueckner E, Song J, et al. Unusual strategies for using indium gallium nitride grown on silicon (111) for solid-state lighting. Proceedings of the National Academy of Sciences. 2011;108(25):10072-10077.

[4] Kim T-H, Cho K-S, Lee EK, et al. Full-colour quantum dot displays fabricated by transfer printing. Nature Photonics. 2011;5(3):176-182.

[5] Cao Q, Kim H-s, Pimparkar N, et al. Medium-scale carbon nanotube thin-film integrated circuits on flexible plastic substrates. Nature. 2008;454(7203):495-500.

[6] Carlson A, Bowen AM, Huang Y, Nuzzo RG, Rogers JA. Transfer printing techniques for materials assembly and micro/nanodevice fabrication. Advanced Materials. 2012;24(39):5284-5318.

[7] Meitl MA, Zhu Z-T, Kumar V, et al. Transfer printing by kinetic control of adhesion to an elastomeric stamp. Nature Materials. 2006;5(1):33-38.

[8] Kim T-H, Carlson A, Ahn J-H, et al. Kinetically controlled, adhesiveless transfer printing using microstructured stamps. Applied Physics Letters. 2009;94(11):113502-3.

[9] Kim S, Carlson A, Cheng H, et al. Enhanced adhesion with pedestal-shaped elastomeric stamps for transfer printing. Applied Physics Letters. 2012;100(17):171909-4.

[10] Carlson A, Kim-Lee H-J, Wu J, et al. Shear-enhanced adhesiveless transfer printing for use in deterministic materials assembly. Applied Physics Letters. 2011;98(26):264104-3.

[11] Carlson A, Wang S, Elvikis P, Ferreira PM, Huang Y, Rogers JA. Active, programmable elastomeric surfaces with tunable adhesion for deterministic assembly by transfer printing. Advanced Functional Materials. 2012;22(21):44764484.

[12] Saeidpourazar R, Li R, Li Y, et al. Laser-driven micro transfer placement of prefabricated microstructures. Journal of Microelectromechanical Systems. 2012;21(5):1049-1058.

[13] Saeidpourazar R, Sangid MD, Rogers JA, Ferreira PM. A prototype printer for laser driven micro-transfer printing. Journal of Manufacturing Processes. 2012;14(4):416-424.

[14] Al-okaily AM, Rogers JA, Ferreira PM. Characterization of delamination in laser microtransfer printing. Journal of Micro and Nano-Manufacturing. 2014;2(1): 011002-1-11.

[15] Li R, Li Y, Lü C, et al. Axisymmetric thermo-mechanical analysis of laser-driven non-contact transfer printing. International Journal of Fracture. 2012;176(2):189-194.

[16] Swinehart D. The Beer-lambert law. Journal of Chemical Education. 1962;39(7):333-335. 
[17] Yang SY, Carlson A, Cheng H, et al. Elastomer surfaces with directionally dependent adhesion strength and their use in transfer printing with continuous roll-to-roll applications. Advanced Materials. 2012;24(16):2117-2122.

\section{Authors' Vitas}

Ala'a M. Al-okaily is currently a graduate student at the department of Mechanical Science and Engineering at the University of Illinois at Urbana-Champaign, where he is pursuing a Ph.D. degree. He received his BS in Industrial Engineering from the Jordan University of Science \& Technology, Jordan followed by MS degree in Manufacturing Systems Engineering from University of Nebraska-Lincoln. His research interests include advanced manufacturing systems and processes, micro- and nano-scale fabrication and integration, precision electro-mechanical systems design, and multi-physics modeling.

Placid M. Ferreira is the Tungchao Julia Lu Professor in Department of Mechanical Science and Engineering, University of Illinois at Urbana-Champaign. He is currently the department head of the Department of Mechanical Science and Engineering at the University of Illinois at Urbana-Champaign. He received his Ph.D. from Purdue University. Professor Ferreira's research focuses on industrial automation, particularly computer-controlled machine tools, nanopositioning and nanosensing, computational geometry and solid modeling as they relate to automated process planning and the discrete-event control of large-scale, flexibly automated systems. 


\section{List of Table Captions}

Table 1. Comparison of different thermo-mechanical FEA models and meshing size for printing $200 \times 200 \times 3 \mu \mathrm{m}$ ink at $10 \mathrm{~A}$ laser current.

\section{List of Figure Captions}

Figure 1 . Examples of uncommon constructs, devices and integrated systems realized by micro transfer printing. (a) SEM image of a printed multilayer stack of silicon platelets [1]. (b) Photograph of a large area $(10 \mathrm{x} 10 \mathrm{~cm})$ negative index metamaterial (NIM) comprised of alternating layers of $\mathrm{Ag}$ and $\mathrm{MgF}_{2}$ in a nano-scale fishnet pattern printed onto a flexible substrate [2]. (c) Photograph of an 'epidermal' electronic device, conformally laminated onto the surface of the skin. (d) Image of a mechanically flexed array of ultrathin, micro-scale, blue LEDs printed from a source wafer onto a thin strip of plastic [3]. (e) Picture of a 4-inch, full-color quantum dot (QD) LED display [4]. (f) Photograph of a flexible integrated circuit that uses printed networks of single walled carbon nanotubes for the semiconductor [5]. (Composite figure taken from $[6])$.

Figure 2. Examples of printing on different surfaces, (left-top) printing on a single $1 \mathrm{~mm}$ ceramic sphere, (middle-top) printing on a non-uniform array of $500 \mu \mathrm{m}$ silica beads, (right-top) printing on to a liquid NOA droplet, (left-bottom) a silicon square printed on to a AFM cantilever, demonstrating assembly on an active structure, (middle-bottom) printing on a ledge, and (right-bottom) printing into recessed spaces. (Scale: in all the micrographs, the printed squares have sides of $100 \mu \mathrm{m}$ ) [8].

Figure 3. A typical laser micro-transfer printing cycle.

Figure 4. Schematic of the laser micro-transfer printer's print head.

Figure 5. A second-generation laser micro transfer printer.

Figure 6. Multi-rays absorption in LMTP process (the solid rays are the ones contributes to the optical absorption while dashed lines are losses rays due to reflection or transmission), the rays are include for demonstration purposes only.

Figure 7. Comparing the power absorbed for different silicon inks thickness at different power levels model estimation with experiments.

Figure 8. Comparing the heating rates for different silicon inks thickness at different power levels model estimation with experiments.

Figure 9. LMTP delamination time experiments at different power levels and ink thicknesses.

Figure 10. 3D model calibration of LMTP based on the post lateral dimensions deformation during printing of $200 \times 200 \times 50$ $\mu \mathrm{m}$ ink at $10 \mathrm{~A}$ laser current, (a) image of the ink-post system at $\mathrm{t}=0 \mathrm{msec}$., (b) image of the ink-post system at delamination start time $\mathrm{t}=9.75 \mathrm{msec}$., (c) 3D model max. lateral deformation (m) at $\mathrm{t}=9.75 \mathrm{msec}$.

Figure 11. Comparison of different thermo-mechanical FEA models ERR values at the ink-stamps interface edge for printing $200 \times 200 \times 3 \mu \mathrm{m}$ ink at $10 \mathrm{~A}$ laser current.

Figure 12. Coupled FEA fields output for printing $200 \times 200 \times 3 \mu \mathrm{m}$ ink at $10 \mathrm{~A}$ laser current, (a) temperature $\left({ }^{\circ} \mathrm{C}\right)$, (b) heat flux in axial direction $\left(\mathrm{W} / \mathrm{m}^{2}\right)$, (c) displacement $(\mathrm{m}),(\mathrm{d})$ axial displacement $(\mathrm{m}),(\mathrm{e})$ mode I strain, (f) mode I stress $(\mathrm{Pa}),(\mathrm{g})$ mode II strain, (h) mode II stress (Pa). 
Figure 13. Interface temperatures estimated from AS model for different inks thicknesses at different power levels, (a) $10 \mathrm{~A}$ current, (b) 15 A current, (c) 20 A current.

Figure 14. Heat fluxes to PDMS post from silicon ink estimated from AS model for different inks thicknesses at different power levels, (a) $10 \mathrm{~A}$ current, (b) $15 \mathrm{~A}$ current, (c) $20 \mathrm{~A}$ current.

Figure 15. ERRs estimated from AS model for different inks thicknesses at different power levels, (a) $10 \mathrm{~A}$ current, (b) $15 \mathrm{~A}$ current, (c) 20 A current.

Figure 16. SIFs at crack tip at the ink-stamp interface estimated from AS model for different inks thicknesses at 10A laser current, (a) mode I SIF, (b) mode II SIF.

Figure 17. SIFs thermal strains decomposition at crack tip at the ink-stamp interface estimated from AS model for $200 \times 200 \times 3 \mu \mathrm{m}$ ink at 10 A laser current, (a) mode I SIF $\left(K_{\mathrm{I}}\right)$, (b) mode II SIF $\left(\mathrm{K}_{\mathrm{II}}\right)$.

Figure 18. SIF's thermal strains decomposition at crack tip at the ink-stamp interface estimated from AS model for different inks thicknesses at 10 A laser current, (a) mode I SIF $\left(\mathrm{K}_{\mathrm{I}}\right)$, (b) mode II SIF ( $\left.\mathrm{K}_{\mathrm{II}}\right)$. 

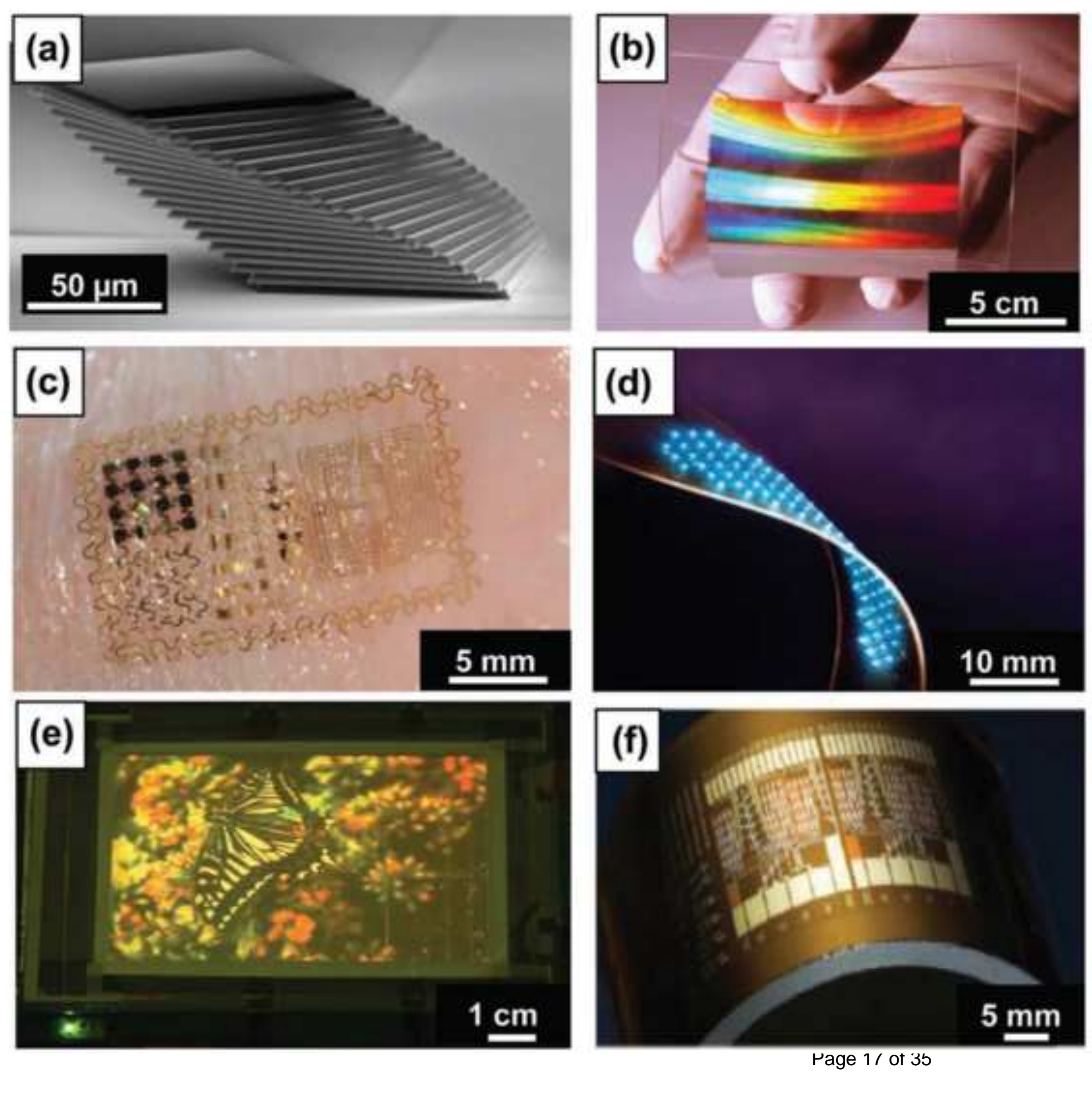

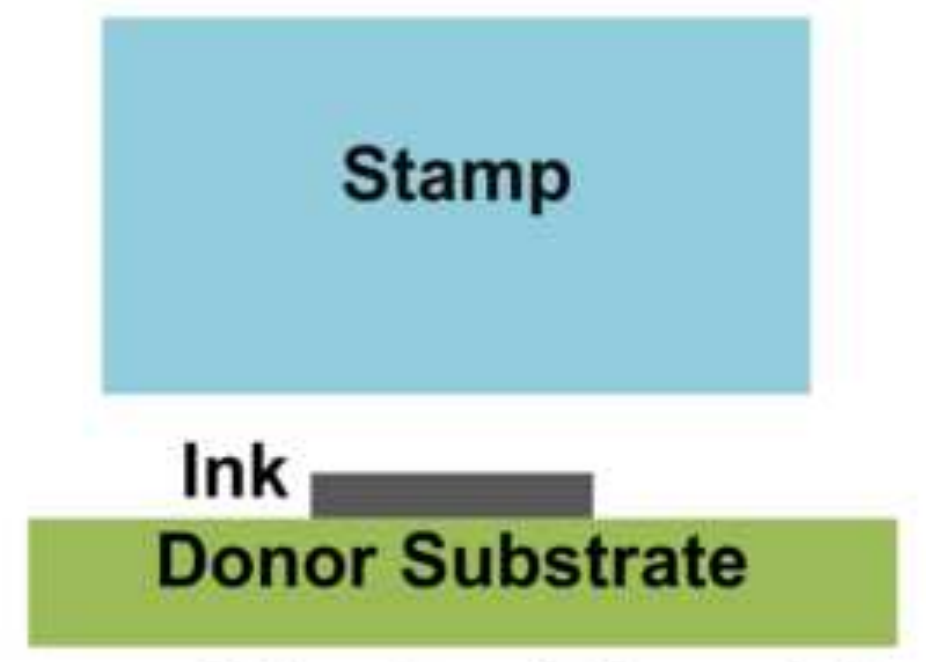

a- Align to pick up ink

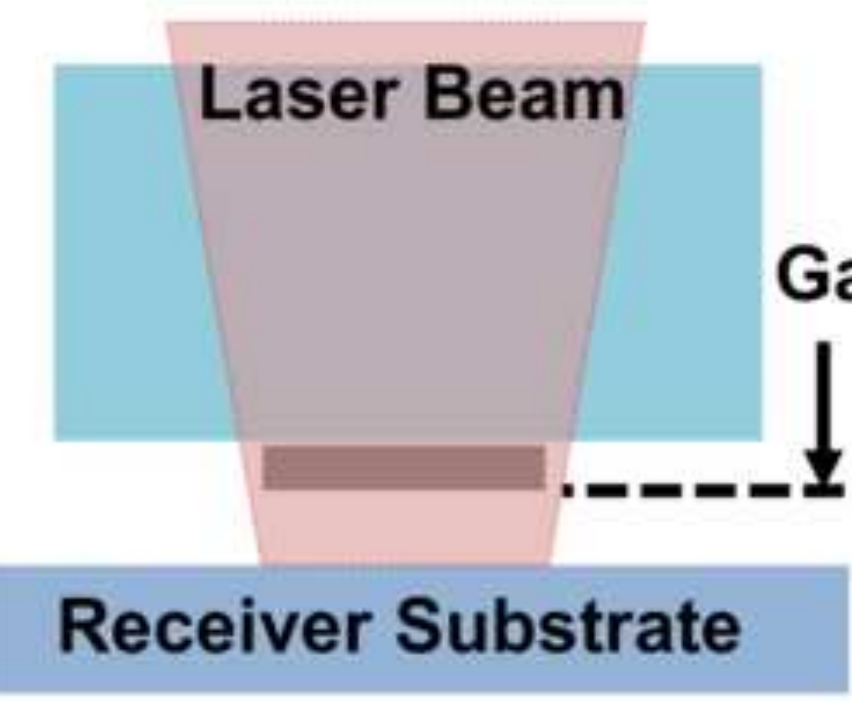

c- Set gap and pulse the laser

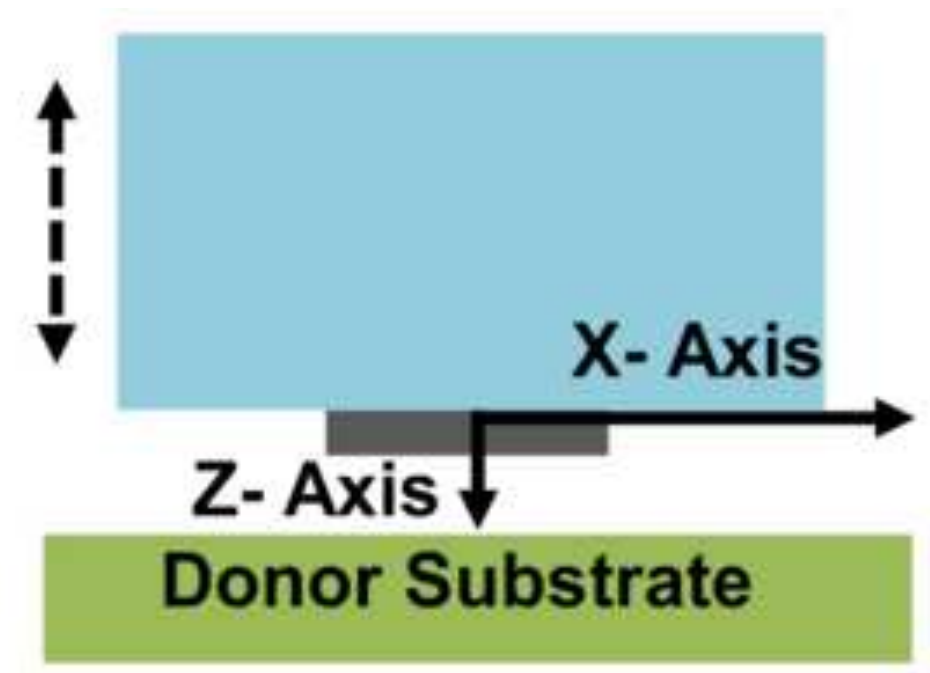

b- Pick up ink

\section{Receiver Substrate}

d- Retract stamp after ink is released 


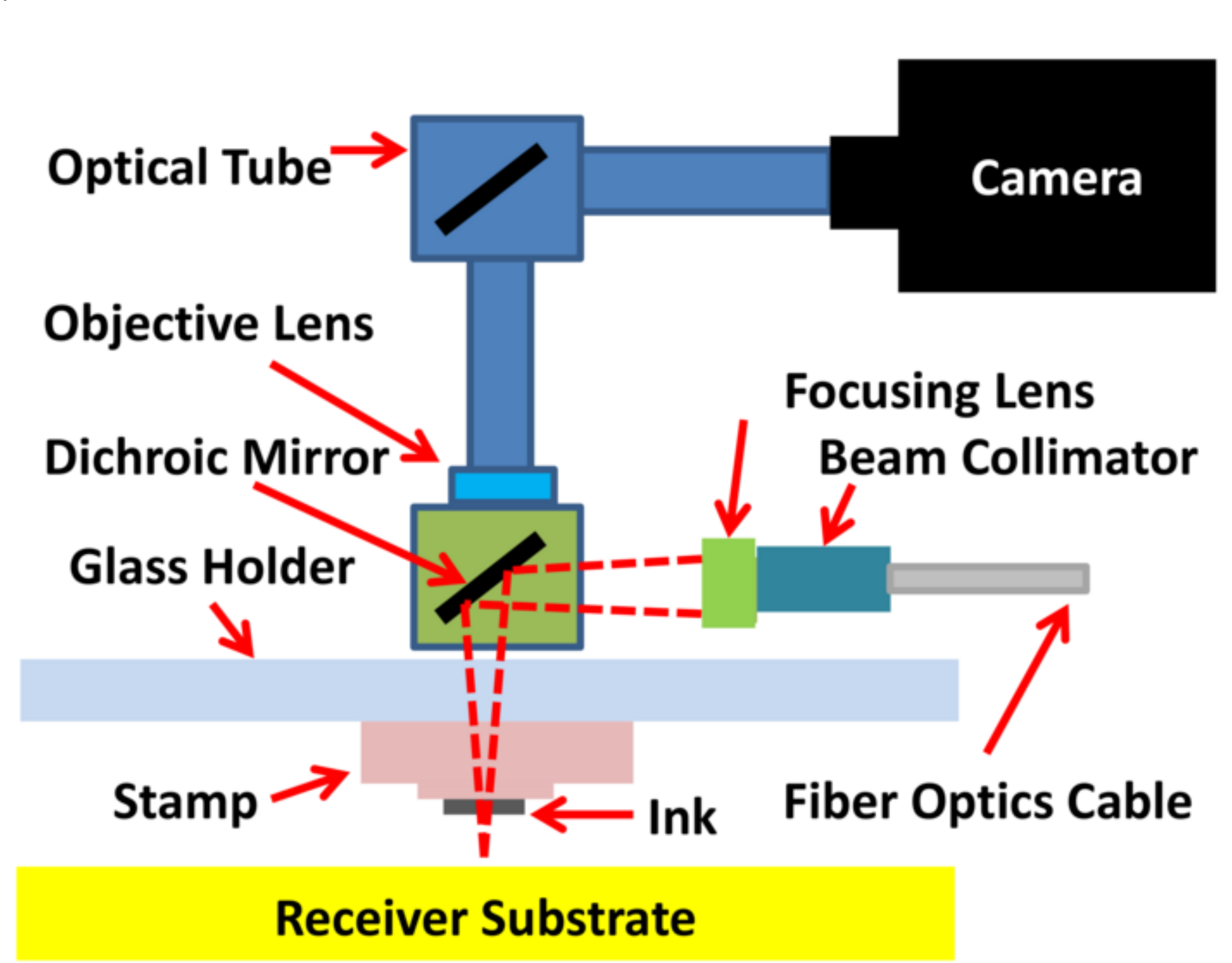

Objective Lens

Dichroic Mirror

Glass Holder

Stamp $\rightarrow$

Focusing Lens Beam Collimator

Fiber Optics Cable

Optical Tube $\rightarrow$

\section{Camera}

rence

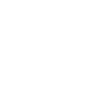




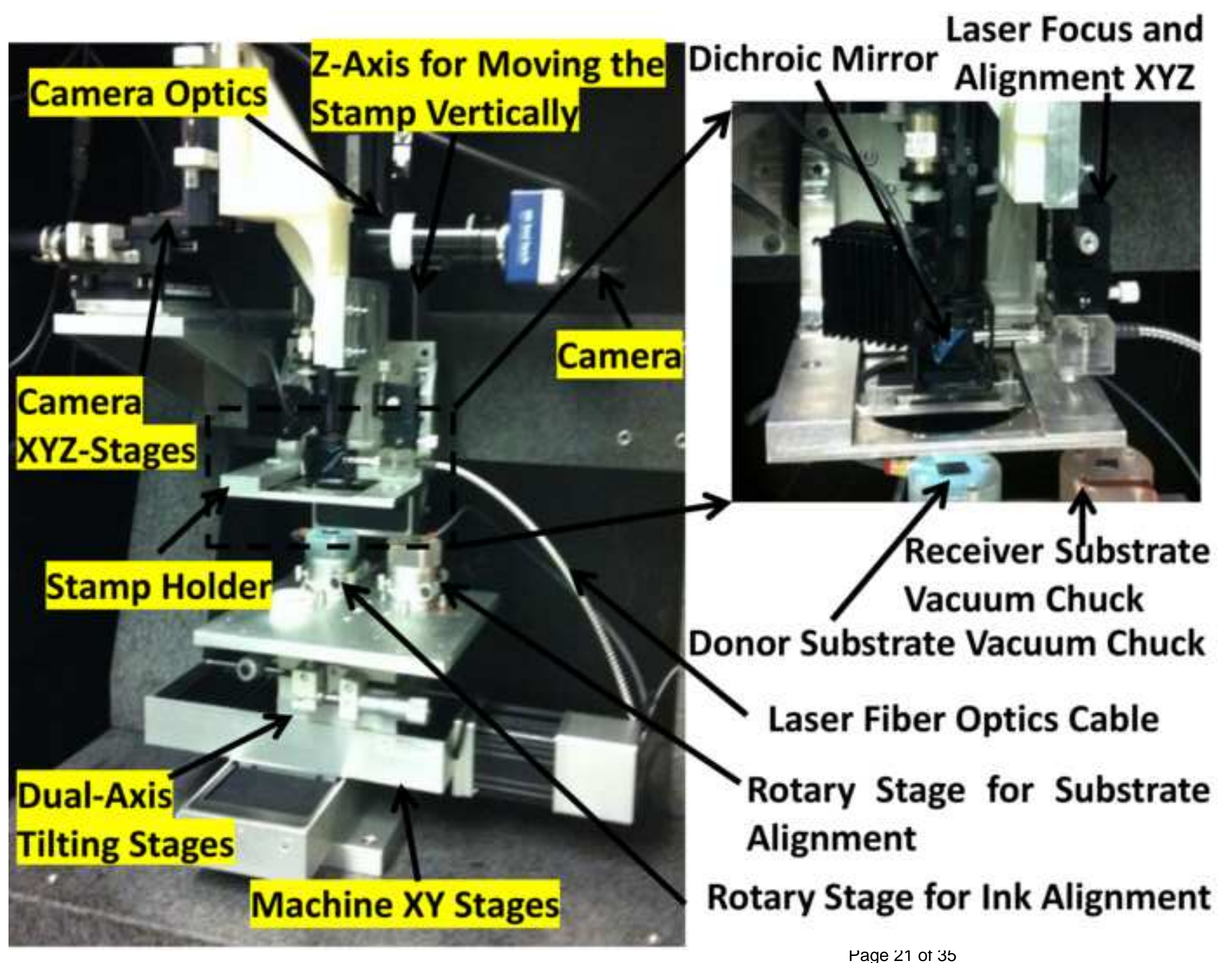

Laser Focus and Alignment XYZ Alignment

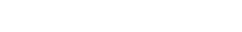

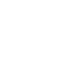

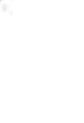

(n)

西




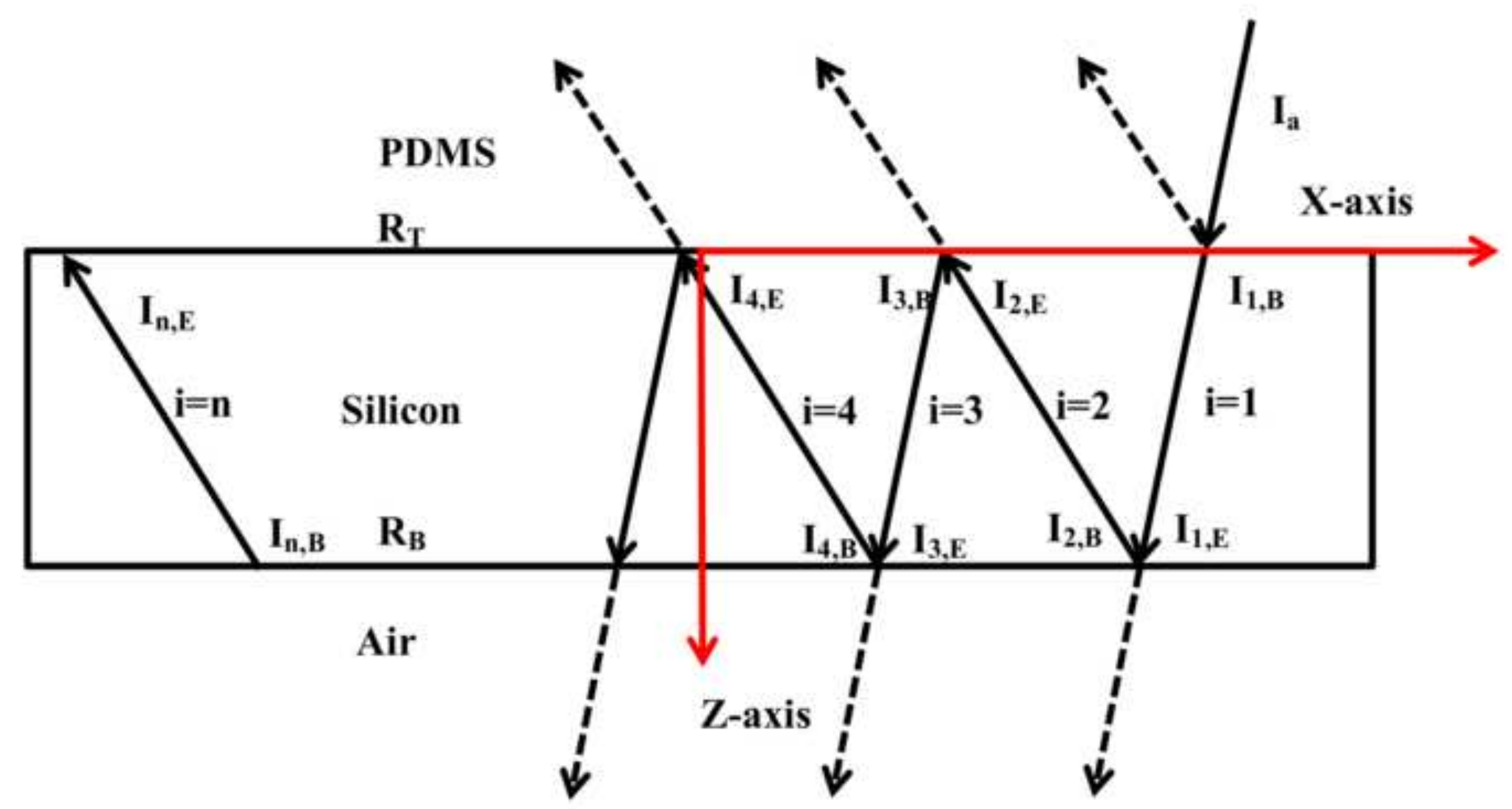




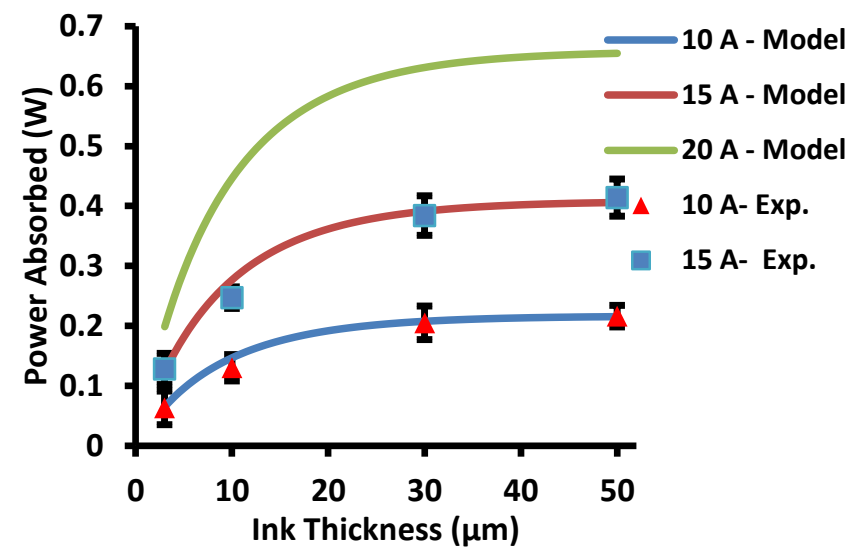




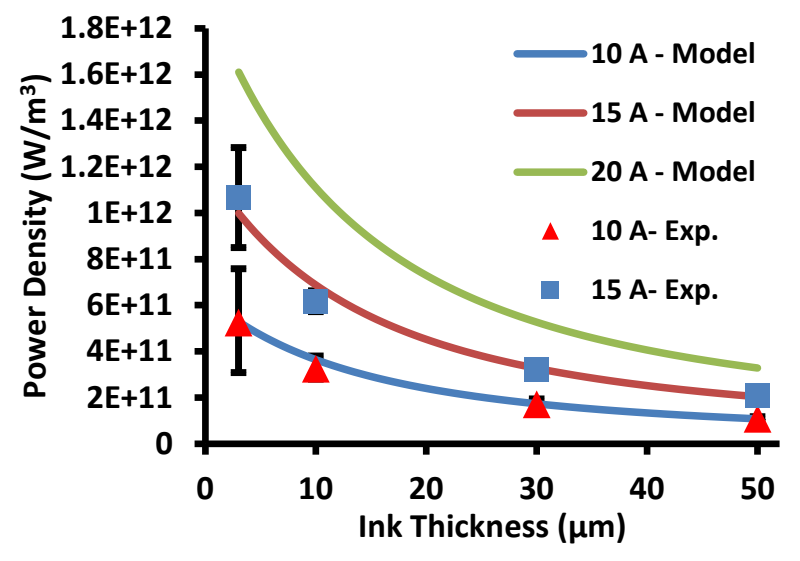

Page 24 of 35 


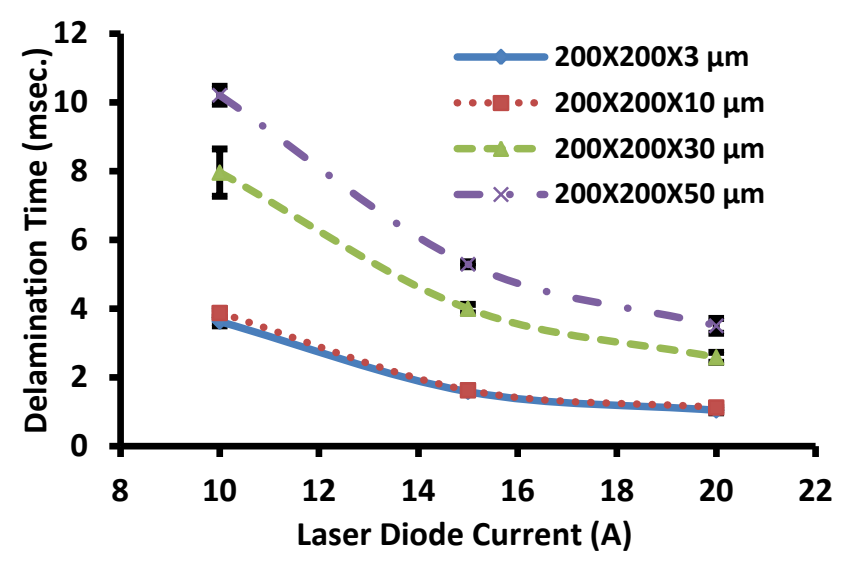

Page 25 of 35 


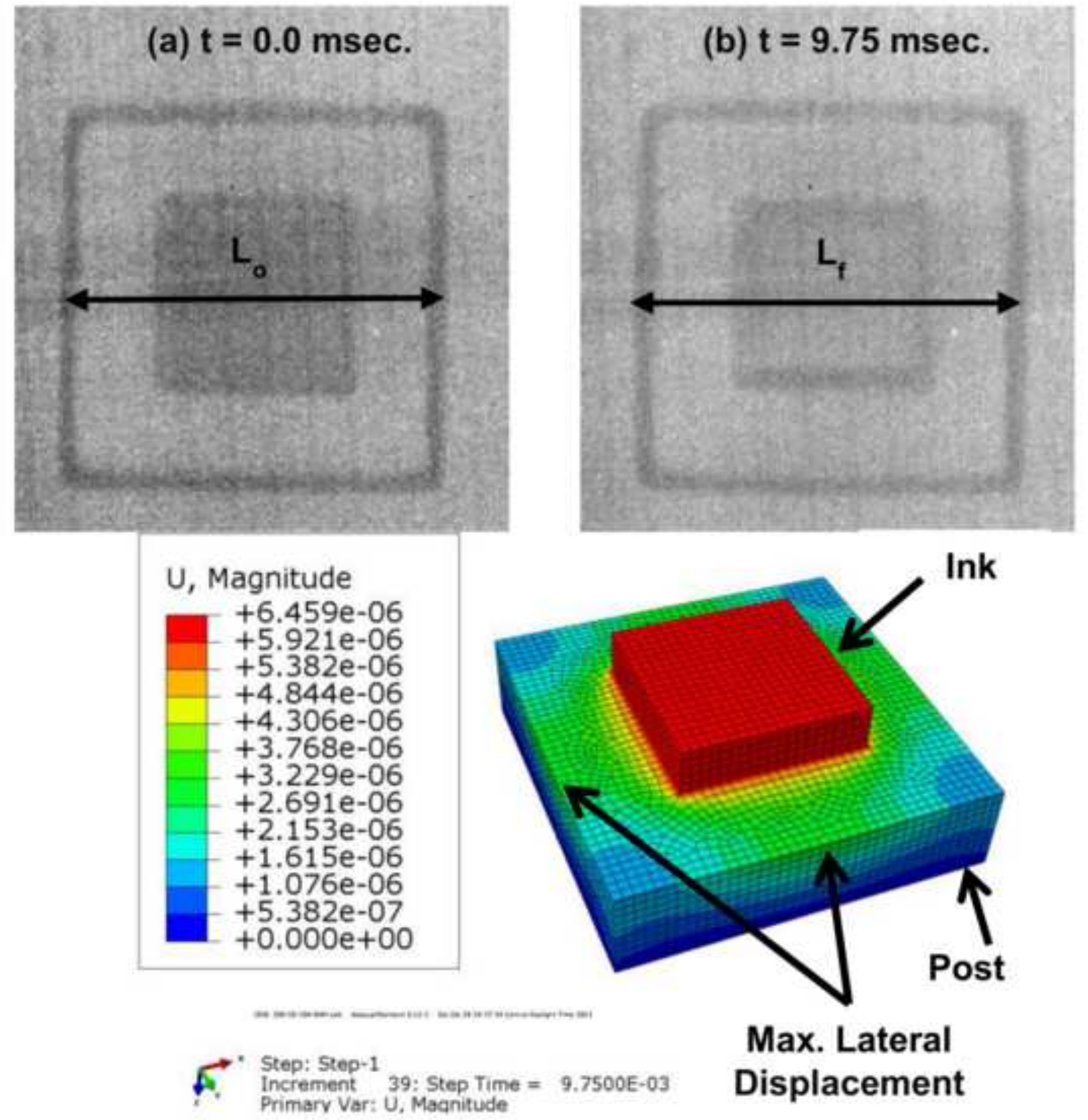

c) FEA model displacement at $\mathbf{t}=9.75 \mathrm{msec}$. 


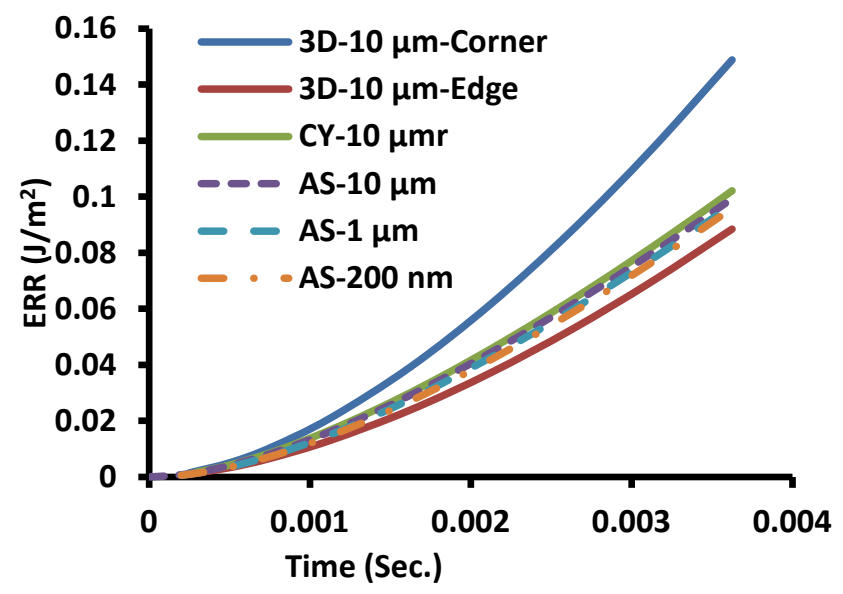

Page 27 of 35 

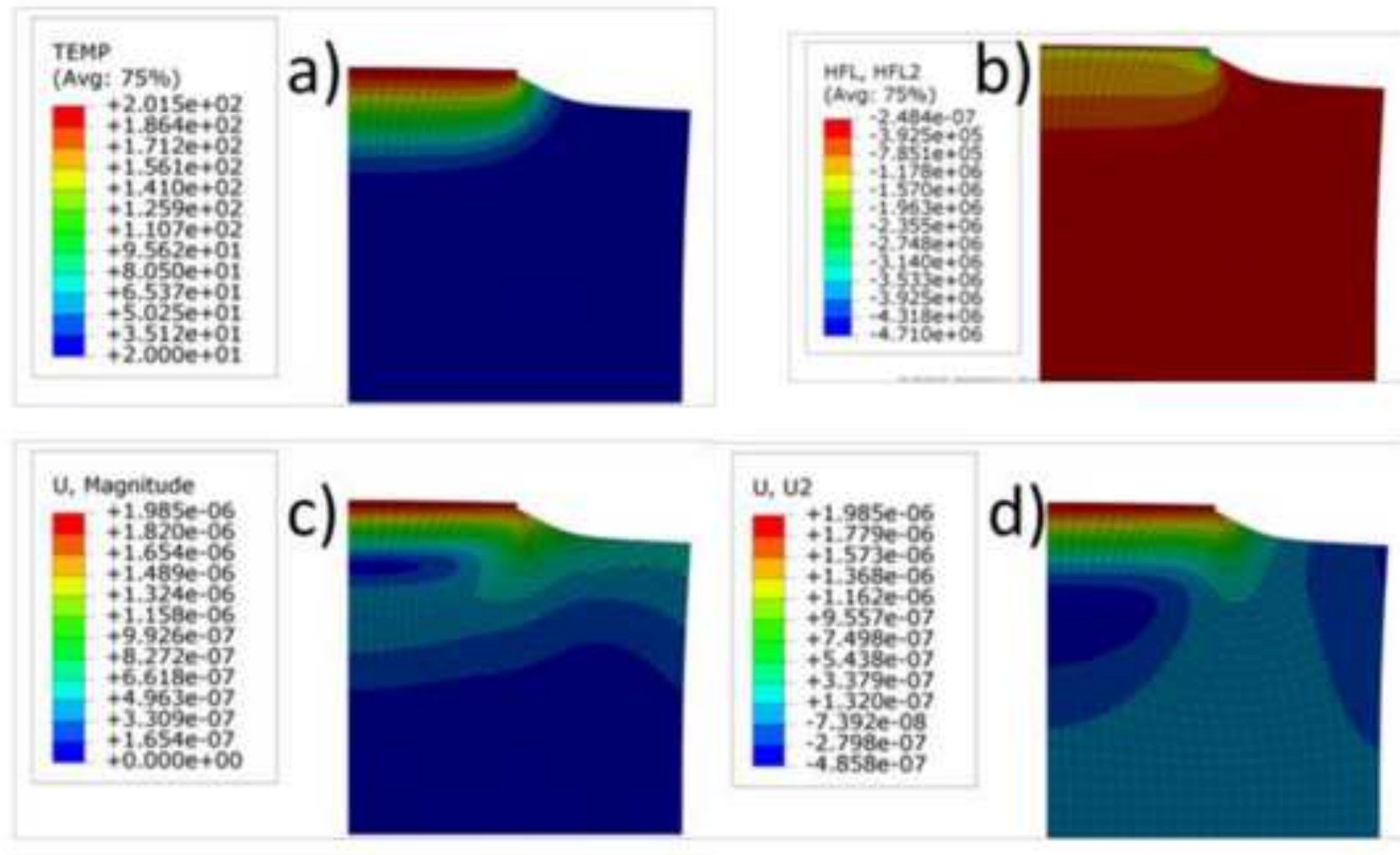

\section{EE, EE22}

(Avg: 75\%)

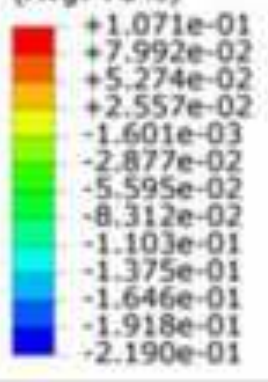

e)

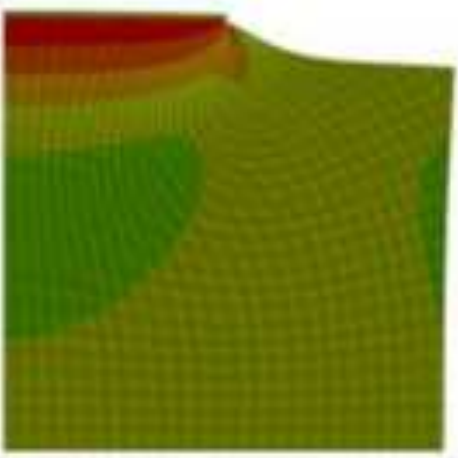

\section{S. 522}

(Avg: 75\%)

$+1327 \mathrm{e}+06$

$1.327 \mathrm{e}+06$
$+1.124 \mathrm{e}+06$ $+9209 \mathrm{e}+05$ $+7.180 \mathrm{e}+05$ $+5.152 e+05$ $+3.124 e+05$ $+1.095 e+05$

$-9,326 e+04$ $-2.961 \mathrm{e}+05$ $-4.969 e+05$ $70180+05$

$9046 e+05$ -1.107e+06 f)

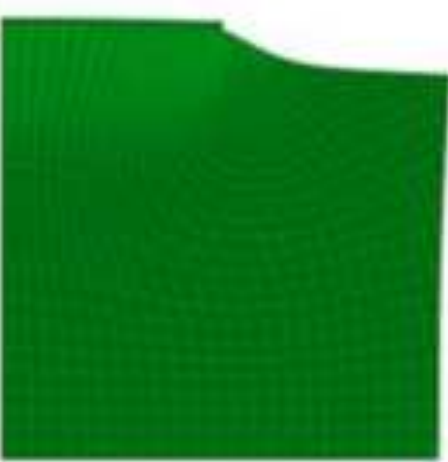

\section{EE. EE12}

(Avg: 75\%)

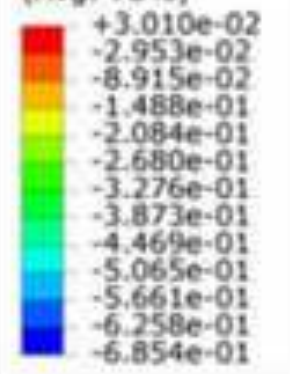

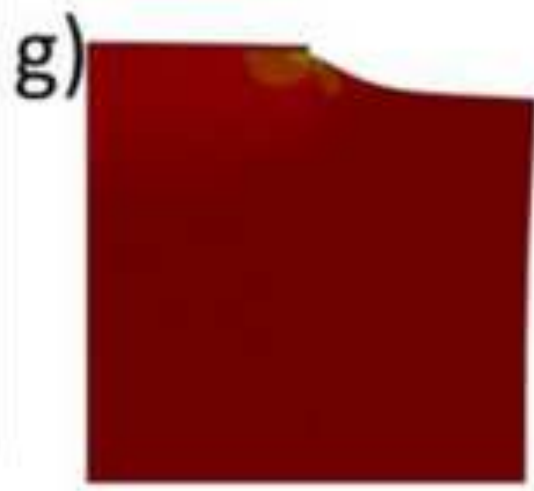

5. $\$ 12$

(Avg: 75\%)

$+1.354 e+05$ $+8.127 e+04$ $+2.714 e+04$ $-2.699 \mathrm{e}+04$ $-8.11\} e+04$ $-1353 e+05$ $-1894 \mathrm{e}+05$ $-2.435 e+05$ -2.977 e+05 $-3518 \mathrm{e}+05$ $-4.059 e+05$ $-4.600 \mathrm{e}+05$ $-5.142 \mathrm{e}+05$

h) 

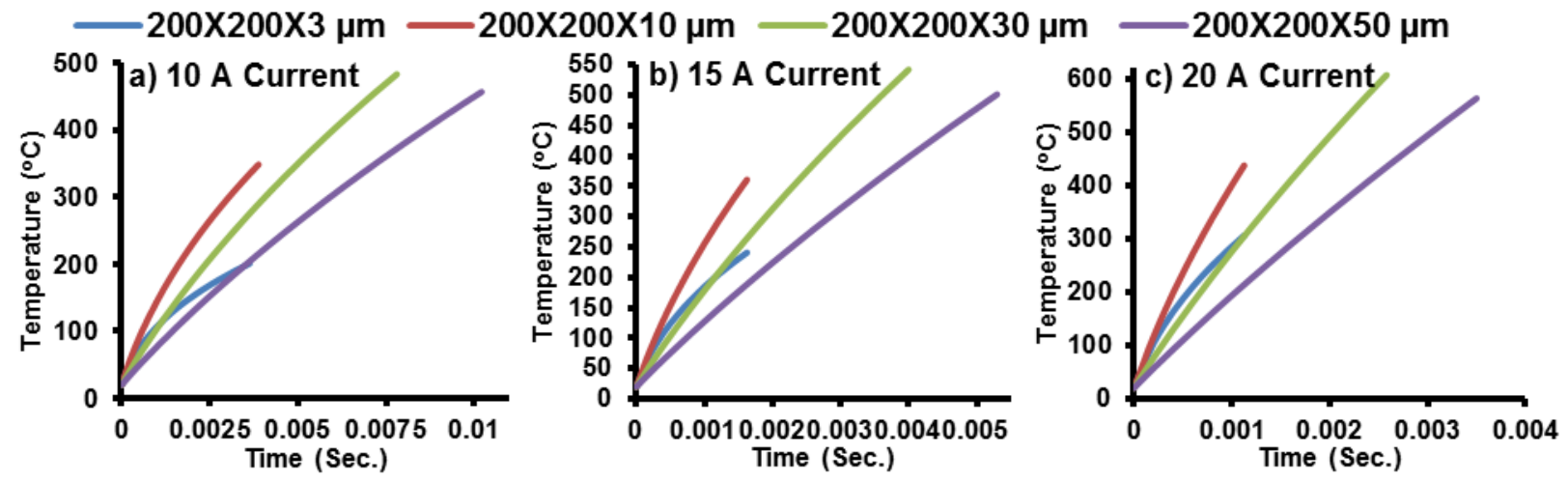

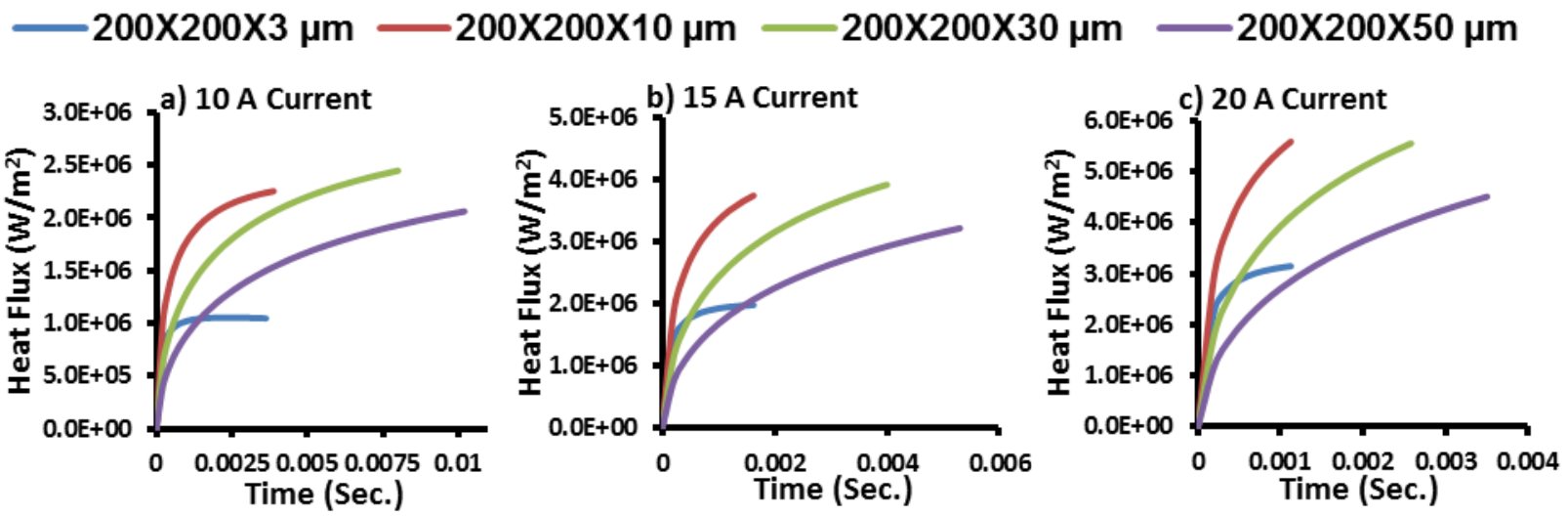

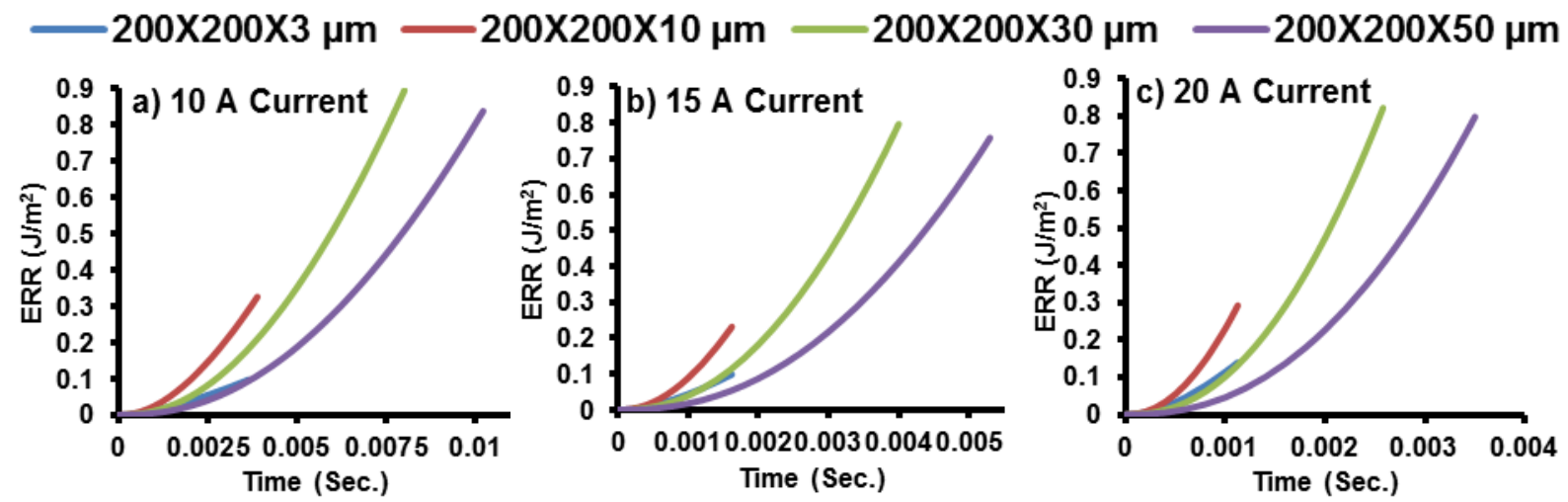

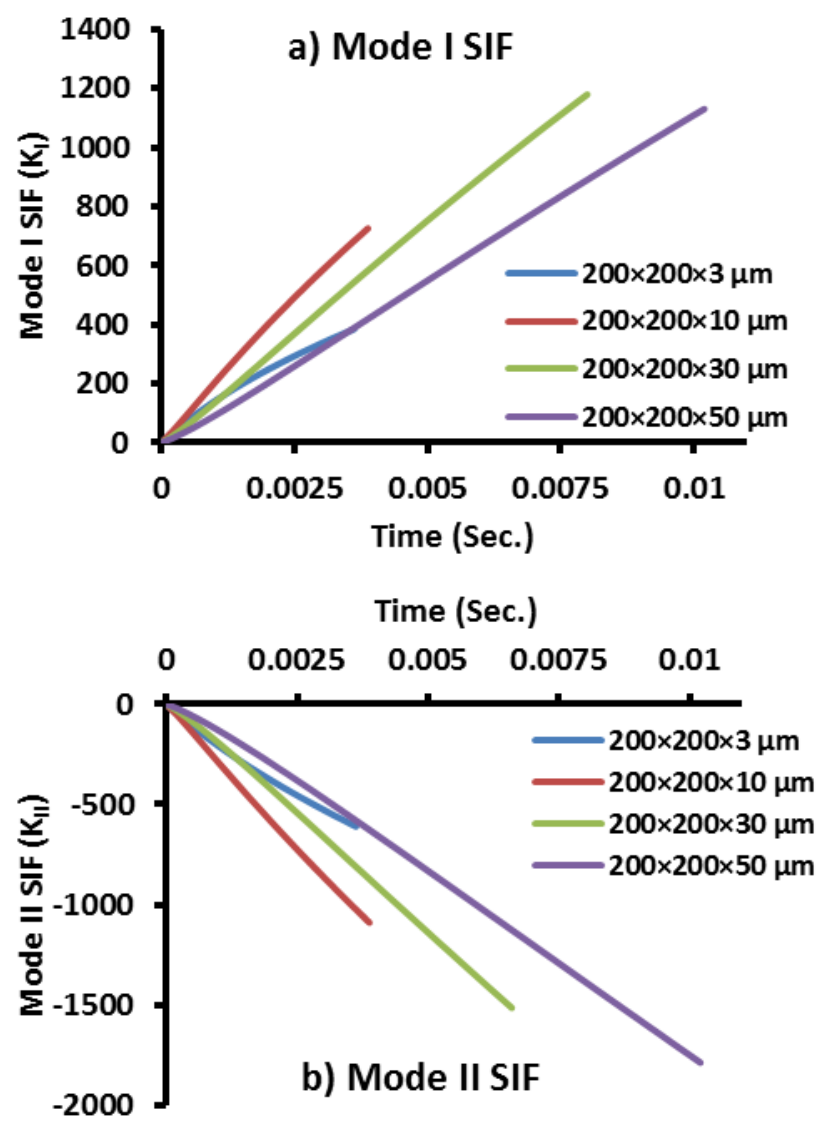

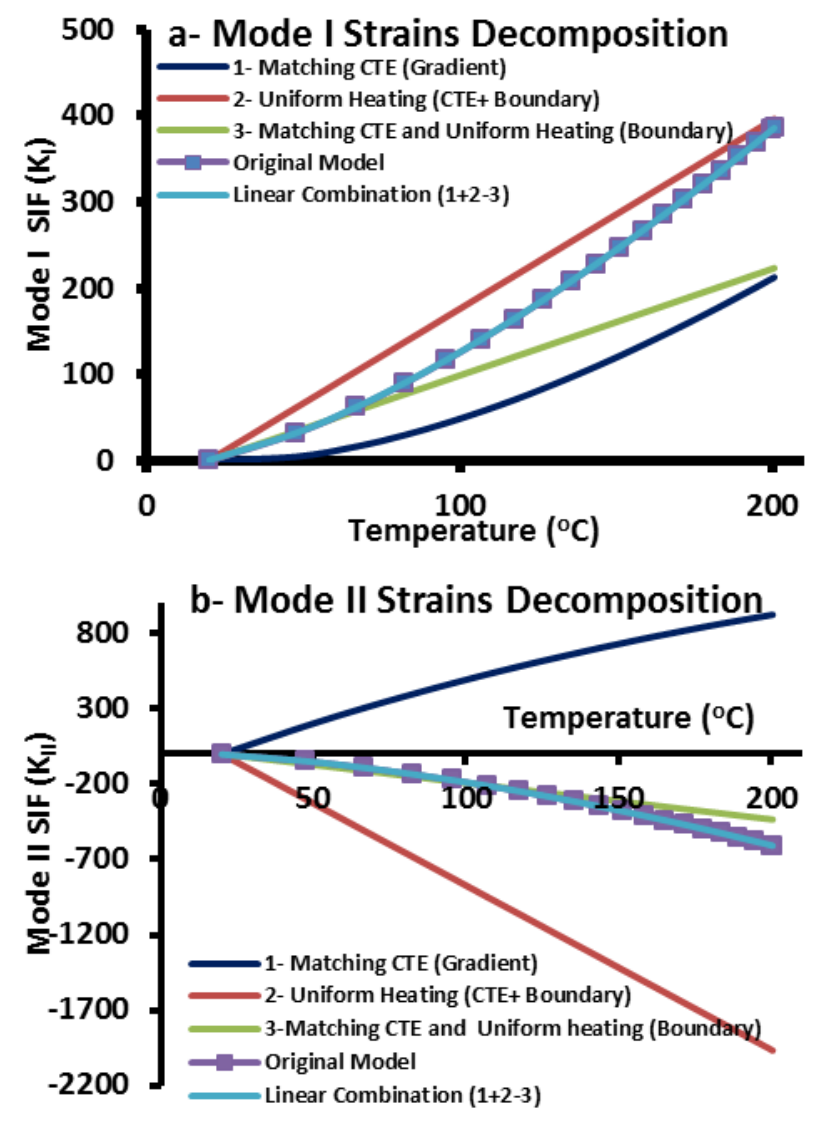

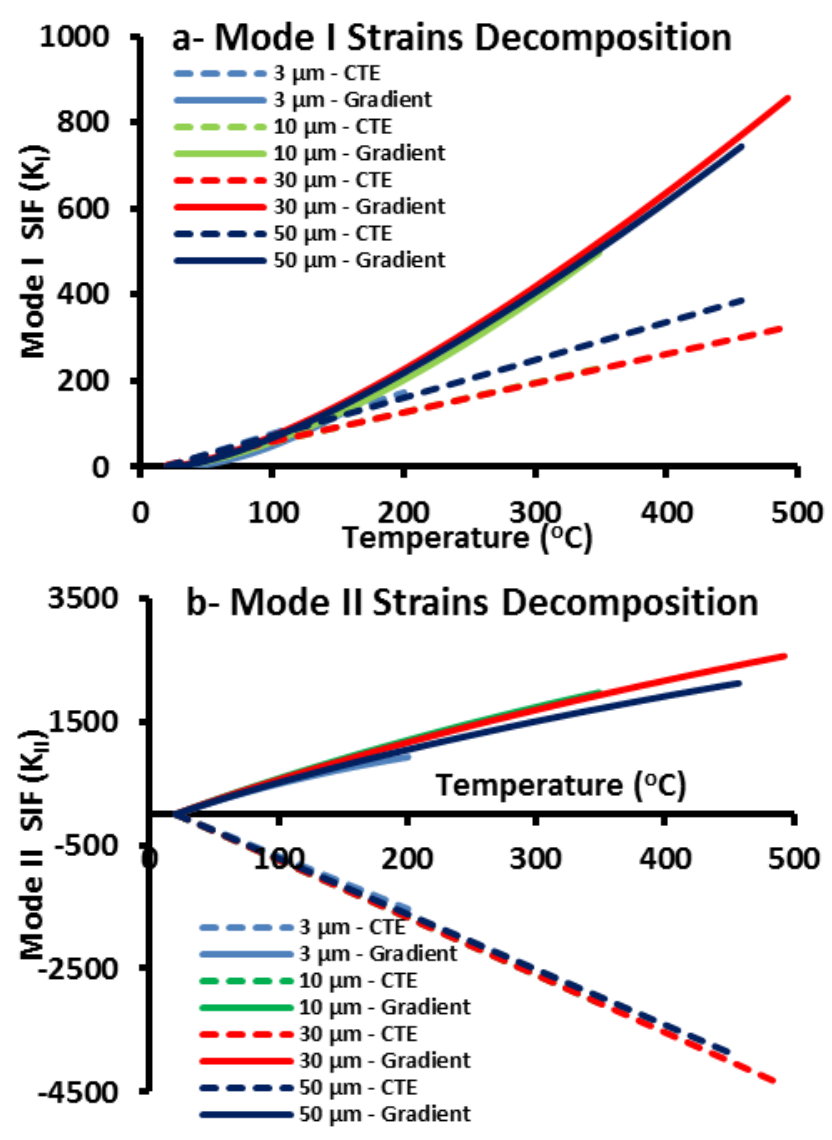


\begin{tabular}{|c|c|c|c|c|c|}
\hline $\begin{array}{l}\text { Model Type } \\
\text { (i.e. Deformed } \\
\text { Temperature Field ) }\end{array}$ & & & & & \\
\hline Global Mesh Size $(\mu \mathrm{m})$ & 10.0 & 10.0 & $\mathbf{1 0 . 0}$ & 1.0 & 0.2 \\
\hline $\begin{array}{l}\text { Interface } \\
\text { Temperature } \quad\left({ }^{\circ} \mathrm{C}\right)\end{array}$ & 171 & 188 & 198 & 201 & 202 \\
\hline $\begin{array}{l}\text { Max. Strain Energy } \\
\text { Density at Ink Edge } \\
\left(\mathbf{J} / \mathbf{m}^{3}\right)\end{array}$ & Corner-2.91 E4 & $1.31 \mathrm{E} 4$ & $2.54 \mathrm{E} 4$ & $1.35 \mathrm{E} 5$ & $6.92 \mathrm{E} 5$ \\
\hline $\begin{array}{l}\text { Max. Stress Mises at } \\
\text { Ink Edge (Mpa) }\end{array}$ & Corner-13.98 & 12.01 & 11.76 & 12.66 & 15.47 \\
\hline $\begin{array}{l}\text { Max. Post Axial } \\
\text { Displacement }(\mu \mathrm{m})\end{array}$ & 2.10 & 2.21 & 1.98 & 1.99 & 2.11 \\
\hline $\begin{array}{l}\text { Max. Post Lateral } \\
\text { Displacement }(\mu \mathrm{m})\end{array}$ & 1.02 & 0.89 & 0.96 & 0.96 & 0.94 \\
\hline $\begin{array}{l}\text { ERR at Ink Edge } \\
\left(\mathrm{J} / \mathbf{m}^{2}\right)\end{array}$ & $\begin{array}{c}\text { Corner-0.149 } \\
\text { Edge- } 0.088 \\
\end{array}$ & 0.102 & 0.099 & 0.097 & 0.096 \\
\hline
\end{tabular}

